\title{
Diffusion Length of Thermal Neutrons in Water
}

\author{
James A. DeJuren and Hyman Rosenwasser
}

\begin{abstract}
The thermal-neutron diffusion length in a hydrogenous medium can be simply determined by measuring the variation of the thermal-neutron density at large radii from the center of a photoneutron source. The necessary requirement is that the relaxation length of the source in a medium be less than the diffusion length, so that diffusion dominates at large radii. Using a $\mathrm{Ra}-\mathrm{Be}$ photoneutron source, the diffusion length of thermal neutrons was determined by indium-foil measurements at large radii. A value of $2.763 \pm 0.015 \mathrm{~cm}$ was obtained. This result was confirmed in a second experiment with an $\mathrm{Sb}^{124}$-Be source.
\end{abstract}

\section{Introduction}

In connection with the National Bureau of Standards program of absolute calibration of a standardneutron source, the diffusion length of thermal neutrons in water was measured. A wide spread of values has been published, and the present experiment is compared with some of the earlier results.

For a high-energy neutron source the thermalneutron density in water at large radii from the source decreases in accordance with the relaxation length defined below. If a source having only low-energy neutrons is employed so that the rate of neutron thermalization per unit volume falls off much more rapidly with increasing radius than the thermalneutron density from a hypothetical thermal-point source, the thermal-neutron density at large radii will vary as a function of the diffusion length and radius only (as in the case of the thermal source). Photoneutron sources have sufficiently low maximum neutron energies for this purpose, and two sources having different neutron-energy maxima were selected for measurements. The general diffusion equation is

$$
d n / d t=q-a+D \nabla^{2} n,
$$

where $n$ is the thermal-neutron density, $q$ is the rate of thermal-neutron production per unit volume (thermal-neutron source-strength density), and $a$ is the absorption rate per unit volume.

$$
a=n v / \lambda_{a} ; \quad D=\lambda_{t r} v / 3,
$$

where $\lambda_{a}$ and $\lambda_{t r}$ are the thermal-neutron absorption and transport mean free paths, respectively, for the average Maxwellian velocity, $v$, and $D$ is the diffusion constant. The steady-state equation is

$$
\nabla^{2} n-n / L^{2}+q / D=0, \quad \text { with } L^{2}=\lambda_{a} \lambda_{t r} / 3,
$$

where $L$ is the diffusion length. This equation has been amply treated by Fermi and others in standard texts.

With water as the medium and at large distances from the source, one can assume with sufficient accuracy that

$$
q=K e^{-r / b} / r^{2},
$$

where $K$ is a constant, $r$ is the distance from the source center, and $b$ is the relaxation length.
This relation is very nearly obeyed even for a source with a broad spectral energy distribution such as is generated by $\mathrm{Ra}=\mathrm{Be}(\alpha, n)$. At large $r, b$ is usually evaluated by measuring resonance activities at energies near thermal with Cd-covered foils. Then at large $r$

$$
\nabla^{2} n-\frac{n}{L^{2}}=\frac{K}{D r^{2}} e^{-r / b}
$$

Since the distribution has spherical symmetry, let $n=u / r$. Then

$$
\nabla^{2} n=\frac{1}{r}\left(\frac{d^{2} u}{d r^{2}}\right)
$$

and

$$
\frac{d^{2} u}{d r^{2}}-\frac{u}{L^{2}}=\frac{K}{D r} e^{-r / b}
$$

The solution to the equation satisfying the boundary condition $u \rightarrow 0$ as $r \rightarrow \infty$ is (derivation in appendix):

$$
u=n r=\frac{K L}{2 D} e^{-\frac{r}{L}}\left[C-\int_{\alpha_{1} r}^{\infty} \frac{e^{-x}}{x} d x+e^{\frac{2 r}{L}} \int_{\alpha_{2} r}^{\infty} \frac{e^{-x}}{x} d x\right],
$$

where $\alpha_{1}=1 / b-1 / L$ and $\alpha_{2}=1 / b+1 / L$. The exponential integrals $\int_{\alpha r}^{\infty}\left(e^{-x} / x\right) d x=E I(-\alpha r)$ may be evaluated from "Tables of sine, cosine and exponential integrals," NBS Mathematical Tables M'T5 and 6 (U. S. Government Printing Office, Washington, D. C., 1947).

As $r \rightarrow \infty$, the solution may be expressed as

$$
n=\frac{c_{1} e^{-r / L}}{r}-\frac{b^{2} L^{2} k}{L^{2}-b^{2}} e^{-r / b} .
$$

If $L>b$, the first term will dominate at sufficiently large radii (the necessary requirement for evaluation of $L$ ).

Since the second term acts as a perturbation, measurements with sources having different values of $b$ serve as a check on the effect of the perturbation at the radii where measurements are possible.

For a $\operatorname{Ra}-\operatorname{Be}(\gamma, n)$ source the maximum neutron energy emitted is only $0.7 \mathrm{Mev}$ (compared to $13 \mathrm{Mev}$ 


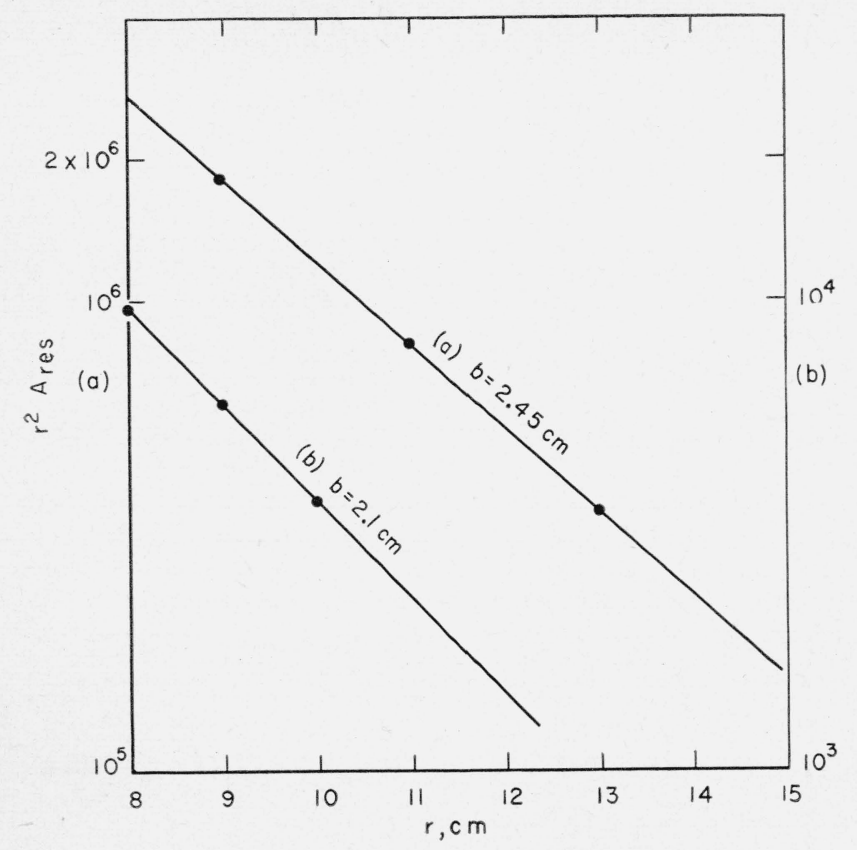

Figure 1. Resonance data for the determination of the relaxation length.

a, $\operatorname{Ra}-\mathrm{Be}(\gamma, n)$ source, indium foils; $\mathrm{b}, \mathrm{Sb}^{124-B e}$ source, gold foils.

for an $\alpha, n$ source), and the slowing-down length is short. Beyond $8 \mathrm{~cm}$ from the center of the National Bureau of Standards photoneutron standard [1] ${ }^{1}$ the indium resonance activity decreases in accordance with eq 3 , with a relaxation length $b=2.45 \mathrm{~cm}$ (fig. 1, a).

\section{Experimental Ārrangement}

The Bureau photoneutron source was placed in the center of a 42-in. diameter stainless-steel tank filled with distilled water. Four indium foils, 2.0 by 3.4 $\mathrm{cm}^{2}$ in area and $76 \mathrm{mg} / \mathrm{cm}^{2}$ thick, held in light aluminum frames, were suspended by wires from a metal framework attached to the top of the tank. The foils were centered in the horizontal plane passing through the source at positions 90 deg apart. A cathetometer mounted horizontally above the tank made it possible to position the foils accurately to within a fraction of a millimeter at the radii employed. The source, a 4-cm-diameter Be sphere enclosing a 1-curie $\mathrm{RaBr}_{2}$ capsule, was pin-mounted on a vertical stainless-steel rod extending from the tank floor.

Periodic measurements of the water temperature gave a mean value of $22^{\circ} \mathrm{C}$, with maximum deviations of $2 \mathrm{deg}$.

\section{Counting Techniques}

The foils were activated to saturation at radii 14 , $16,18,20$, and $22 \mathrm{~cm}$ from the origin with and without 0.020 -in. Cd covers. All four foils were simultane-

\footnotetext{
1 Figures in brackets indicate the literature references at the end of this paper.
}

ously counted with "thin" mica end-window Geiger counters, the outputs of which were fed to a mixing circuit, which permitted the foils to be counted as a unit by a single scaler. The sides of the foils facing the source also faced the counter windows when placed in their respective holders inside the lead Geiger-counter shields. Uranium standards were used to monitor the Geiger counters, and repetitive cycles were made of standard, background, and foils, with counting intervals of 5,10 , and 20 minutes, respectively, during the decay of the foils.

\section{Results}

The data are given in table 1 . A correction of 6 percent must be made for the absorption of $1.44 \mathrm{ev}$ In resonance neutrons by the $\mathrm{Cd}$ covers. Errors given are determined by the consistency of the data as well as the counting statistics. The activities given are the counting rates of the foils at saturation normalized to the same monitor reading. Figure 2 is a semilog plot of radius times thermal activity as a

TABLE 1. Data from NBS standard Ra-Be photoneutron source

\begin{tabular}{|c|c|c|c|c|}
\hline & $\begin{array}{c}\text { Activity } \\
\text { Position } \\
(r \text { in } \mathrm{cm})\end{array}$ & $\begin{array}{c}\text { Resonance } \\
\text { (Counts } \\
\text { per minute) } \\
\text { activity } \times \\
1.06 \text { (Counts } \\
\text { per minute) }\end{array}$ & $\begin{array}{c}\text { Thermal } \\
\text { activity } \\
\left(A_{t h}\right) \\
\text { (Counts } \\
\text { per minute) }\end{array}$ & $r A_{t h}$ \\
\cline { 1 - 1 } 14.0 & $7,657 \pm 38$ & $190 \pm 7$ & $7,467 \pm 39$ & 104,540 \\
16.0 & $3,546 \pm 18$ & $64 \pm 15$ & $3,482 \pm 23$ & 55,710 \\
18.0 & $1,533 \pm 9$ & a $22 \pm 6$ & $1,511 \pm 11$ & 27,200 \\
20.0 & $655 \pm 10$ & a $8 \pm 3$ & $647 \pm 10$ & 12,940 \\
22.0 & $292 \pm 5$ & a $3 \pm 1$ & $289 \pm 5$ & 6,360 \\
\hline
\end{tabular}

\& Calculated.

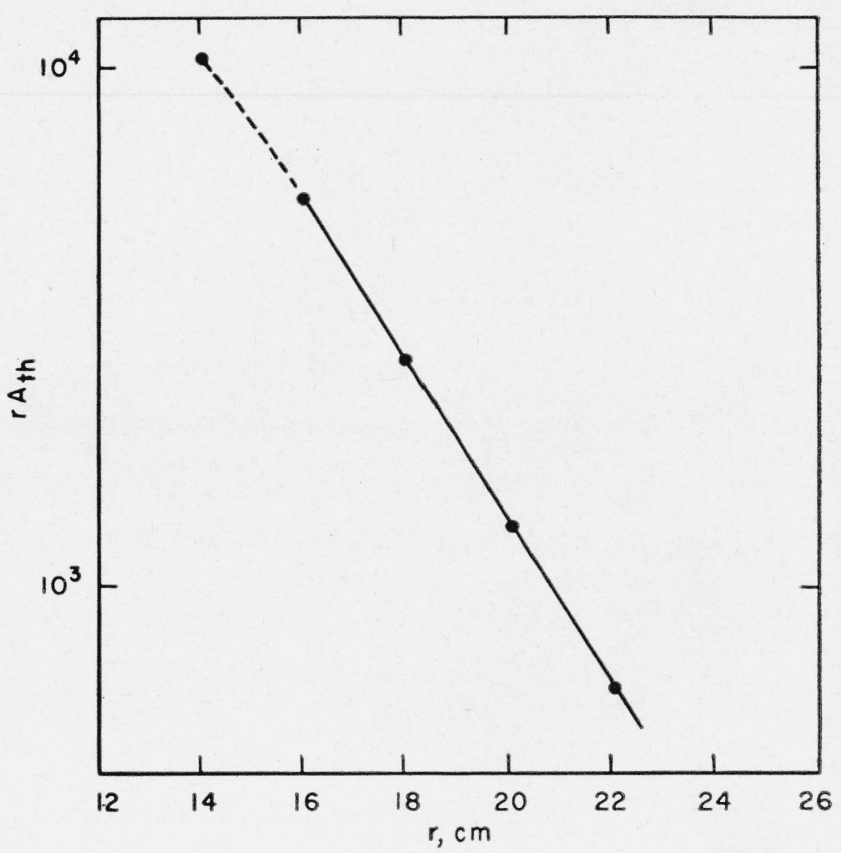

Figure 2. Indium foils in water.

Rः-Be $(\gamma, n)$ source. 
function of $r$. Beyond $16 \mathrm{~cm}, r A_{t h}$ decreases exponentially.

$$
\frac{A_{1} r_{1}}{A_{2} r_{2}}(1 \pm \epsilon)=e \frac{r_{2}-r_{1}}{L}
$$

where $\epsilon$ is the experimental error of the ratio

$$
\ln \left(\frac{A_{1} r_{1}}{A_{2} r_{2}}\right) \pm \epsilon=\frac{r_{2}-r_{1}}{L} .
$$

Using the data at 16 and $22 \mathrm{~cm}$ as an example,

$$
\begin{gathered}
\ln \frac{5571}{636} \pm \epsilon=\frac{6}{L} \\
2.170\left(1 \pm \frac{\epsilon}{2.170}\right)=\frac{6}{L} ; \quad \epsilon=0.018 \\
L=2.765 \pm 0.023 \mathrm{~cm} .
\end{gathered}
$$

The weighted least-squares fit of the data between 16 and $22 \mathrm{~cm}$ yield $L=2.763 \pm 0.015 \mathrm{~cm}$.

\section{5. $\mathrm{Sb}^{124}$-Be Experiment}

An antimony pellet that was irradiated in the Oak Ridge National Laboratory pile for 2 months was substituted for the $\mathrm{RaBr}_{2}$ capsule in a 4 -cm-diameter beryllium sphere. The 60 -day $\mathrm{Sb}^{124}$ has three $\gamma$-rays above the Be photoneutron threshold, the principal one having an energy of $1.7 \mathrm{Mev}$ and giving rise to 24-kev neutrons [2]. As the neutron energies are considerably lower than those from the NBS standard source, moderation occurs closer to the source, and

\begin{tabular}{|c|c|c|c|c|}
\hline $\begin{array}{l}\text { Position } \\
(r \text { in } \mathrm{cm})\end{array}$ & $\begin{array}{c}\text { Activity } \\
\text { without Cd } \\
\text { (Counts } \\
\text { per minute) }\end{array}$ & $\begin{array}{l}\text { Resonance } \\
\text { activity } \times \\
1.06 \text { (Counts } \\
\text { per minute) }\end{array}$ & $\begin{array}{c}\text { Thermal } \\
\text { activity } \\
\left(A_{t h}\right) \\
\text { (Counts } \\
\text { per minute) }\end{array}$ & $r A_{t h}$ \\
\hline $\begin{array}{l}14.0 \\
16.0 \\
18.0 \\
20.0\end{array}$ & $\begin{aligned} 625.8 & \pm 6 \\
262.3 & \pm 10 \\
110.7 & \pm 6 \\
50.6 & \pm 5\end{aligned}$ & $\begin{array}{r}12.4 \pm 3.0 \\
\mathrm{a} 1.5 \pm 0.5 \\
\mathrm{a} 0.5 \pm 0.2 \\
\mathrm{a} .2 \pm 0.1\end{array}$ & $\begin{aligned} 613.4 & \pm 7 \\
260.8 & \pm 10 \\
110.2 & \pm 6 \\
50.4 & \pm 5\end{aligned}$ & $\begin{array}{l}8,588 \\
4,173 \\
1,984 \\
1,008\end{array}$ \\
\hline
\end{tabular}
the relaxation length is shorter. A value of $2.1 \mathrm{~cm}$

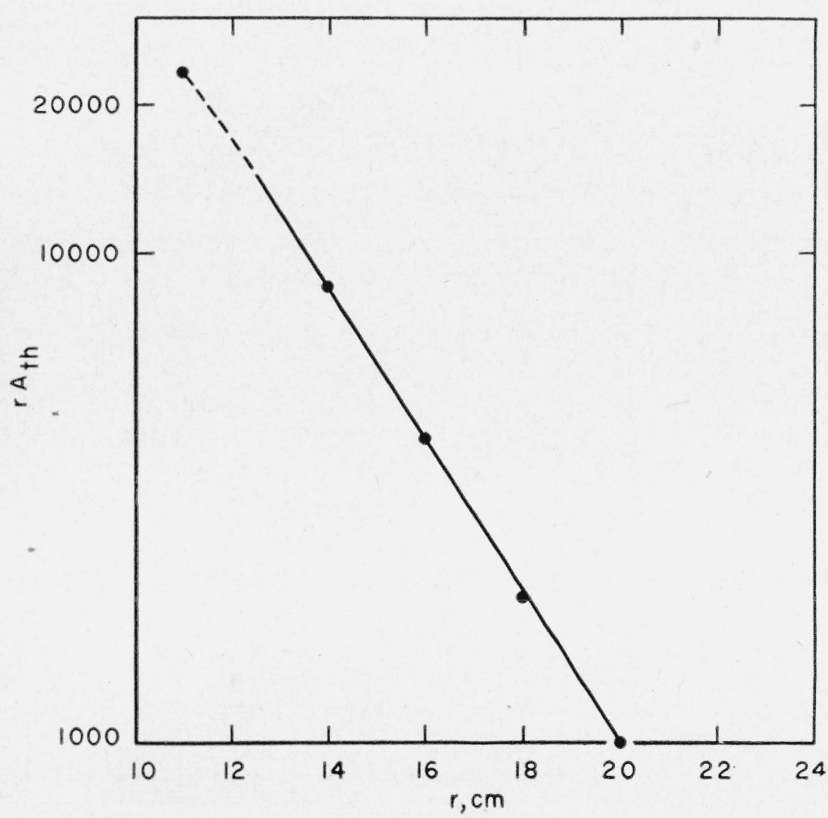

Figure 3. Indium foils in water.
TABLE 2. Data from $\mathrm{Sb}^{124}$-Be photoneutron source

a Calculated.

for $b$ was obtained from the Sb-Be source in water, the measurements being made with $\mathrm{Cd}$-covered indium and gold foils at radii beyond $8 \mathrm{~cm}$ from the source (fig. 1,b).

Indium foils that had been intercalibrated in the same slow neutron flux were simultaneously exposed to saturation at different radii from the source. The foils were successively removed and counted with the same end-window tube. For radii greater than 12 $\mathrm{cm}, r A_{t h}$ decreases exponentially. At $14 \mathrm{~cm}$ the corrected cadmium reading is 1 percent of the thermal reading and decreases to 0.35 percent at $20 \mathrm{~cm}$ (calculated by using $b=2.1$ ). The least-squares fit of the data (table 2 ) gives a value of $L$ equal to $2.76 \pm 0.07$ (fig. 3), which is in agreement with the $\mathrm{Ra}-\mathrm{Be}$ result, even though the ratio of indium resonance to thermal-neutron counting rates is considerably lower for the $\mathrm{Sb}-\mathrm{Be}$ over the range covered.

\section{Discussion and Comparison of Results}

For a purely thermal source located at the origin

and

$$
n(r)=C e^{-r / L} / r
$$

$$
\overline{r^{2}}=\frac{\int_{0}^{\infty} n(r) r^{4} d r}{\int_{0}^{\infty} n(r) r^{2} d r}=6 L^{2}
$$

In analogy, the slowing-down distance, $L_{s}$, of neutrons from a high-energy source is usually estimated from the mean-square distance obtained from resonance-neutron measurements. $\overline{r_{r e s}^{2}}=6 L_{s}^{2}$, and the approximate relation

$$
\overline{r_{\text {thermal }}^{2}}=\overline{r_{\text {res. }}^{2}}+6 L^{2}
$$

is used to calculate $L^{2}$.

Equation (7) treats the resultant thermal-neutron distribution as though the high-energy neutrons diffused from the source with a diffusion length equal to $\sqrt{L_{s}^{2}+L^{2}}$. The shape of the radial thermal and resonance-neutron density distributions are actually Gaussian near the origin. As a result, the thermal and resonance $\overline{r^{2}}$ values give somewhat overestimated diffusion lengths, and the value of $L$ obtained by this method should represent an upper bound to the true figure. 
Table 3 lists some of the early values obtained with $\operatorname{RaBe}(\alpha, n)$ sources, using eq 7 .

TABLE 3.

\begin{tabular}{|c|c|c|c|c|c|}
\hline Detector & $\overline{r_{\text {thermal }}^{2}}$ & $\overline{r_{\text {res. }}^{2}}$ & $L$ & $b$ & $L_{s}$ \\
\hline $\begin{array}{l}\text { Rh [3] } \\
\text { In [4] } \\
\text { Dy }+ \text { In }[5] \\
\text { In [6] } \\
\text { In [7] }\end{array}$ & $\begin{array}{l}c m{ }^{2} \\
327 \\
337 \\
330 \\
371 \\
363\end{array}$ & $\begin{array}{l}c m^{2} \\
277 \\
272 \\
278 \\
288 \\
313\end{array}$ & $\begin{array}{l}c m \\
2.85 \\
3.3 \\
2.94 \\
3.6 \\
2.88\end{array}$ & $\begin{array}{r}c m \\
9.4 \\
9.4 \\
10.1 \\
9.4 \\
9.8\end{array}$ & $\begin{array}{l}c m \\
6.8 \\
6.7 \\
6.8 \\
6.9 \\
7.2\end{array}$ \\
\hline
\end{tabular}

Since eq 7 involves a small difference between two numbers, a 1 percent error in either $\overline{r^{2}}$ term causes about a 3-percent error in $L$.

Berthelot, Cohen, and Reel [8] have determined $L$ with a $\operatorname{Ra}-\operatorname{Be}(\alpha, n)$ source in a water tank by a different technique. A thin spherical shell, which could be filled alternately with $\mathrm{H}_{2} \mathrm{O}$ (or $\mathrm{H}_{2} \mathrm{O}+\mathrm{H}_{2} \mathrm{SO}_{4}$ ) and a $\mathrm{CdSO}_{4}$ solution, surrounded the source. The difference in the neutron densities outside the sphere for the two cases arises from the diffusion of thermal neutrons present when the sphere is filled with water. Measurements with foils gave a best value of $2.77 \pm$ $0.04 \mathrm{~cm}$ for the thermal-diffusion length.

Larsson [9] recently has made extensive measurements of the same type at different $\mathrm{CdSO}_{4}$ concentrations. An attempt was made to correct for the perturbation in the slow-neutron density in the immediate vicinity of the sphere and for the attenuation of high-energy neutrons by the cadmium. He obtained a diffusion length of $3.25 \pm 0.13 \mathrm{~cm}$. However, a different interpretation of the data would give a lower value.

Perhaps the simplest method for measuring $L$ is afforded by a nuclear reactor. If a water tank is placed on top of a thermal column, the neutron density, a few transport mean free paths from the boundary should decrease as $e^{-Z / L}$, providing that the diameter and height of the tank are much greater than the diffusion length. Early reactor experiments gave a value of about $2.8 \mathrm{~cm}$. Sisk [10] has recently obtained a value of $2.67 \pm 0.02 \mathrm{~cm}$ at $24^{\circ} \mathrm{C}$, using the Oak Ridge pile and different boundary conditions.

Probably most of the variation in the values of $L$ obtained by the different techniques is due to the failure to meet experimentally the rigorous boundary conditions required.

In the present experiment an upper limit to $L$ is obtained unless the bracketed term in eq 5 can be determined. The exponential integrals have been evaluated, using $L=2.76$, and $C$ was roughly determined for the radium source by calibrating the foils in a standard flux. A perturbation of less than 5 percent $(C>1.3)$ in $L$ should be caused by neglecting the exponential integrals. For the $\mathrm{Sb}-\mathrm{Be}$ source, the exponential integrals are much smaller, and neglecting them should affect the calculation of $L$ less. The $\mathrm{Sb}-\mathrm{Be}(\gamma, n)$ source was fairly weak, and a stronger source would permit measurements beyond $20 \mathrm{~cm}$, where the exponential integrals would have negligible effect.
The advice and cooperation of L. F. Curtiss is gratefully acknowledged, as well as the assistance of R. N. Olcott, D. Padgett, and W. Bailey in obtaining the experimental data of this paper. We are indebted to A. N. Gleyzal for assistance in the solution of the diffusion equation.

\section{References}

[1] L. F. Curtiss and A. Carson, Phys, Rev. 76, 1412 (1949).

[2] K. Way, Nuclear Data, NBS Circular 499 (1950).

[3] E. Amaldi and E. Fermi, Phys. Rev. 50, 899 (1936).

[4] J. H. Rush, Phys. Rev. 73, 271 (1948).

[5] Munn and Pontecorvo, Can. J. Research 25A, 157 (1947).

[6] C. W. Tittle, MIT Ph. D. Thesis, January 1948.

[7] J. Dacey, R. Paine, and C. Goodman, unpublished report.

[8] Berthelot, Cohen and Reel, Compt. rend. 225, 406 (1947).

[9] Larsson, Arkiv. Fysik, 2, 47 (1950).

[10] F. J. Sisk, Oak Ridge National Laboratory Report 933 (1951).

\section{Appendix}

Solution of diffusion equation:

Let $p=\frac{\partial}{\partial r}$

$$
\frac{d^{2} u}{d r^{2}}-\frac{u}{L^{2}}=-\frac{K}{D r} e^{-r / b}
$$

$$
\begin{aligned}
& (p-1 / L)(p+1 / L) u=-\frac{K}{D r} e^{-r / b} \\
& u=\frac{-1}{(p-1 / L)(p+1 / L)} \frac{K}{D r} e^{-r / b}
\end{aligned}
$$

Solving by partial fractions:

$$
\begin{gathered}
\frac{1}{(p-1 / L)(p+1 / L)}=\frac{S_{1}}{p-1 / L}+\frac{S_{2}}{p+1 / L} \\
S_{1}=L / 2, \quad S_{2}=-L / 2 \\
u=L K / 2 D\left[\frac{1}{p+1 / L}-\frac{1}{p-1 / L}\right] \frac{e^{-r / b}}{r} \\
u=C_{1} e^{-r / L}+C_{2} e^{+r / L}+K L / 2 D \\
{\left[e^{-r / L} \int \frac{e^{-r(1 / b-1 / L)}}{r} d r-e^{r / L} \int \frac{e^{-r(1 / b+1 / L)}}{r} d r\right] .}
\end{gathered}
$$

Let $\alpha_{1}=1 / b-1 / L, \alpha_{2}=1 / b+1 / L$.

The indefinite integrals may be expressed in terms of definite exponential integrals

$$
\int \frac{e^{-\alpha r}}{r} d r=-\int_{r}^{\infty} \frac{e^{-\alpha y}}{y} d y=-\int_{\alpha r}^{\infty} \frac{e^{-x}}{x} d x .
$$

To satisfy boundary conditions at infinity $C_{2}=0$, and setting $C_{1}=K L C / 2 D$.

$$
u=n r=\frac{K L}{2 D} e^{-r / L}\left[C-\int_{\alpha_{1} r}^{\infty} \frac{e^{-x}}{x} d x+e^{2 r / L} \int_{\alpha_{2} r}^{\infty} \frac{e^{-x}}{x} d x\right]
$$

To evaluate $C, K$ must be determined. Since less than 1 percent of the neutrons from either source are captured above thermal energies

$$
Q=4 \pi \int_{r_{0}}^{\infty} q(r) r^{2} d r=4 \pi \int_{r_{0}}^{\infty}-K e^{-r / b} d r
$$


where $Q$ is the neutron emission rate of the source.

$$
Q=4 \pi b K e^{-r_{0} / b} \text {. }
$$

The expression $q=K e^{-r / b} / r^{2}$ does not hold down to the radius of the source, so a larger value of $r_{0}$ than the source radius must be used. For the $\operatorname{Ra}-\operatorname{Be}(\gamma, n)$ source $r_{0}$ is estimated to be between 4.0 and $4.5 \mathrm{~cm}$ from previous data. Using $K=Q e^{r_{0} / b} / 4 \pi b$, an absolute measurement of $n$ would permit a rough determination of $C$ as all other quantities are approximately known.

Exponential integrals for $L=2.76$ :

\begin{tabular}{|c|c|c|}
\hline Source & $\alpha_{1}$ & $\alpha_{2}$ \\
\hline $\begin{array}{l}\mathrm{Ra}-\mathrm{Be} \\
\mathrm{Sb}-\mathrm{Be}\end{array}$ & $\begin{array}{l}0.0458 \\
.114\end{array}$ & $\begin{array}{r}0.7705 \\
.8385\end{array}$ \\
\hline
\end{tabular}

\begin{tabular}{|c|c|c|}
\hline \multicolumn{3}{|c|}{$\mathrm{Ra}-\mathrm{Be}(\gamma, n)$ source } \\
\hline$r$ & $\int_{\alpha_{1} r}^{\infty} e^{-x} / x d x$ & $e^{2 r / L} \int_{\alpha_{2} r}^{\infty} e^{-x / x} d x$ \\
\hline $\begin{array}{l}\mathrm{cm} \\
16 \\
180 \\
22\end{array}$ & $\begin{array}{r}0.351 \\
.297 \\
.253 \\
.217\end{array}$ & $\begin{array}{r}0.037 \\
.030 \\
.024 \\
.019\end{array}$ \\
\hline \multicolumn{3}{|c|}{$\mathrm{Sb}-\mathrm{Be}(\gamma, n)$ source } \\
\hline $\begin{array}{l}14 \\
16 \\
18 \\
20\end{array}$ & $\begin{array}{r}0.0868 \\
.0625 \\
.0455 \\
.0339\end{array}$ & $\begin{array}{r}0.0161 \\
.0113 \\
.0078 \\
.0054\end{array}$ \\
\hline
\end{tabular}

Washington, July 1, 1953. 